Paidéia, 2005, 15(32), 417-425

\title{
CONTEXTO E NORMAS DE ASSOCIAÇÃO PARAPALAVRAS: A REDUÇÃO DO CAMPO SEMÂNTICO ${ }^{1}$
}

Gerson Américo Janczura²

IP/PPB - Universidade de Brasília

\begin{abstract}
Resumo: Este estudo teve por objetivo coletar normas de associação semântica para categorias naturais. Utilizando-se o método da associação livre, os participantes foram solicitados a produzir a primeira associada que completasse o significado de sentenças que serviam como contexto para as categorias. As associações produzidas em dois contextos semânticos diferentes foram comparadas com associações produzidas sem contexto. As seguintes medidas foram produzidas para cada categoria: o tamanho da categoria, as associadas semânticas, erros, omissões e respostas idiossincráticas. Propôs-se, ainda, um índice de ocorrência intracategórica que permite identificar quais são os membros mais provavelmente lembrados, independentemente da condição experimental. A análise das normas evidenciou um efeito de redução do campo semântico, e identificou os membros a serem gerados, apesar de a categoria ter sido apresentada isoladamente ou em contextos diferentes. Uma redução média de cinqüenta por cento no campo semântico foi observada quando as categorias conceituais estão contextualizadas.
\end{abstract}

Palavras-chave: normas de associação; memória semântica; categorias naturais.

\section{CONTEXT AND NORMATIVE DATA FOR WORDS: THE SEMANTIC REDUCTION EFFECT}

\begin{abstract}
This study presents semantic association norms for natural categories. Participants were instructed to produce the first response to sentence cues using a free association procedure. The free associations produced in two different contexts were contrasted to categories presented with no context. The following parameters were calculated: category set size, word associates, errors, omissions and idiosyncratic responses. A measurent index that indicates which category members are more likely remembered regardless of category condition was proposed. Results indicated a reduction effect on the semantic field. It was also demonstrated that some category members are more likely produced regardless of whether the category was experienced either with or without context. The category semantic field is reduced about fifty per cent when categories are presented within a sentence context.
\end{abstract}

Key words: word norms; semantic memory; natural categories.

Introdução: As palavras têm sido amplamente utilizadas na investigação e compreensão dos processos cognitivos, um dos estímulos preferidos pelos pesquisadores em função da facilidade de sua manipulação, familiaridade dos indivíduos com este material e sua participação cotidiana nas atividades humanas. Estima-se que as pessoas conheçam, em média, 50.000 palavras (Barsalou, 1992).

\footnotetext{
${ }^{1}$ Artigo recebido em 28/04/2005, aceito para publicação em 20/09/2005. 2 Endereço para correspondência: Gerson Américo Janczura Laboratório de Processos Cognitivos, Universidade de Brasília, Campus Darcy Ribeiro, Brasília/DF, CEP: 70910-900, E-mail: janczura@unb.br.
}

Entre os atributos das palavras, que têm despertado o interesse na pesquisa sobre a aprendizagem, memória e cognição, encontra-se a semântica, a sintaxe, o comprimento, a freqüência de ocorrência em materiais escritos, a imagística, a afetividade, a concretude, a similaridade acústica, a tipicidade, a ressonância, a conectividade, a familiaridade, a homografia, o número de palavras associadas, a força de associação entre elas, e a acessibilidade na memória. A manipulação sistemática destes atributos tem contribuído para compreensão da memória, recuperação e reconhecimento (Nelson, 


\section{Gerson Américo Janczura}

McKinney, Gee \& Janczura, 1998), representação mental de conceitos (Hampton \& Gardiner, 1983), escuta dicótica (Eling, 1983), fenômeno da ponta-da-língua (Kozlowsky, 1977), fala e linguagem (Bishop \& Robson, 1989), processos desenvolvimentais (Wiegel-Crump \& Dennis, 1986), envelhecimento e memória (Shaw \& Craik, 1989), processamento semântico (Lupiáñes, Rueda, Ruz \& Tudela, 2000), afetividade e memória (Elmes, Dye \& Herdelin, 1983; Jacoby \& Dallas, 1981).

A utilização de palavras na pesquisa impõe uma seleção cuidadosa destes estímulos porque seus atributos podem produzir, se não apropriadamente controlados, efeitos indesejáveis de confusão. A necessidade de evitar a possibilidade de influências secundárias demanda conhecimento sobre os atributos das palavras. Um dos procedimentos frequentemente utilizado na identificação dos atributos é a coleta de normas, o que já acontece na Psicologia há mais de 50 anos. Alguns exemplos são as normas sobre a freqüência absoluta de ocorrência de palavras de Thorndike e Lorge (1944) e de Kucera e Francis (1967), as de concretude, imagística e significação de Paivio, Yuille e Madigan (1968), as para categorias semânticas de McEvoy e Nelson (1982) e Battig e Montague (1969), para rimas de palavras de Libkuman (1994), de tipicidade e familiaridade para itens verbais de Hampton e Gardiner (1983) e Marques (1997), para associação livre entre palavras de Nelson, McEvoy e Schreiber (1999), Hirsh e Tree (2001) e Macizo, Gómez-Ariza e Bajo (2000), as de familiaridade, complexidade visual e nomeação de Snodgrass e Vanderwart (1980) e de Pérez e Navalón (2003), e de força associativa intra-categórica de Callejas, Correa, Lupiáñez e Tudela (2003).

Apesar de normas estarem sendo utilizadas há muito tempo na Psicologia, no Brasil poucos são os estudos que as usam em função, provavelmente, da escassez deste tipo de ferramenta. $\mathrm{O}$ pesquisador brasileiro dispõe das normas de freqüência de ocorrência de palavras em material impresso de Pinheiro (1996) e Sardinha (2004), as de associação semântica para palavras de Janczura (1996), as de associação de palavras para o paradigma DRM de Stein e Pergher (2001), que é uma versão traduzida e adaptada das normas de Stadler, Roediger e McDermott (1999), e as de fragmentos de palavras para o teste de Completação de Radicais de Palavras de Pompéia, Paes e Bueno (2003).
Normas de freqüência de ocorrência em materiais impressos são úteis nos estudos sobre a recuperação e reconhecimento, tendo-se observado que este fator produz um efeito dissociativo entre os respectivos paradigmas experimentais. Além disto, a freqüência está associada com o tempo na tarefa de decisão léxica (Balota, Ferraro e Conor, 1991), interage com a repetição (Nelson, McEvoy e Bajo, 1984) e com o intervalo de retenção (Hulme, Roodenvys, Schweickert, Brown, Martin e Stuart, 1997). Normas de associações semânticas para palavras são ferramentas indispensáveis na pesquisa sobre as memórias implícita e explícita, falsas memórias, representação e aprendizagem de conceitos, raciocínio dedutivo, influências do envelhecimento na aprendizagem e cognição, entre outros. Ilustrando, Stein e Pergher (2001) mostraram que falsas memórias podem ser produzidas utilizando-se o paradigma DRM, que inclui palavras associadas semanticamente. Janczura e Nelson (1999) mostraram que a força associativa de um exemplo, em relação a sua categoria semântica, pode prever a percepção de sua tipicidade conceitual. Além disto, atributos da estrutura associativa matricial das palavras na memória influenciam a evocação com pista e o reconhecimento (Nelson, McKinney, Gee e Janczura, 1998).

Normas de associação para palavras estimam o conjunto de associadas em virtude de alguma propriedade ou relação lingüística e permitem o mapeamento de campos léxicos, proporcionando uma forma de avaliar o conhecimento sobre palavras que os indivíduos adquiriram durante a sua história de aprendizagem (Nelson, Shreiber e McEvoy, 1992). Esta abordagem difere das concepções clássicas do significado das palavras como a teoria da referência, a da imagem ou a semântica formal (Barsalou, 1992).

Uma das tarefas mais utilizadas na coleta das associadas de uma palavra é a associação livre. Neste procedimento um conjunto de palavras é apresentado para grandes amostras de indivíduos, que devem produzir a primeira palavra (método da primeira resposta) que lhes vier à mente para cada uma das palavras da lista. Usualmente, as normas que têm coletado o campo semântico, usando esta técnica, apresentam as palavras isoladamente. Isto é, as palavras-alvo não estão condicionadas a qualquer contexto que possa influenciar a produção de associadas 
gráfica, fonêmica ou semântica. Experimentalmente, o contexto tem sido definido como qualquer informação (uma palavra, sentença ou texto) apresentada junto com os estímulos a serem processados, de alguma maneira, segundo instruções solicitadas pelo pesquisador. No caso das normas de associação semântica, algumas interpretações são mais prováveis do que outras dependendo das condições em que as palavras são apresentadas (isto é, sem ou com contexto).

No presente estudo, normas de associação semântica foram coletadas para categorias conceituais inseridas no contexto de sentenças. A tarefa dos participantes era produzir uma associada (isto é, um membro da categoria conceitual) que se encaixasse nas respectivas sentenças. Nesta condição, as normas oferecem a oportunidade de avaliar o comportamento semântico de palavras contextualizadas em contraste com a ausência de informações que possam delimitar o campo semântico, como é o caso das coletadas por Janczura (1996). É esperado que o contexto delimite o campo semântico das palavras, entretanto, ainda não foi demonstrada a magnitude deste efeito para palavras apresentadas nas duas condições. O objetivo deste estudo foi avaliar o respectivo efeito em três situações: na ausência de contexto e em dois contextos diferentes. Um dos parâmetros produzidos através deste tipo de procedimento é o tamanho do conjunto de associadas (número de palavras produzidas na tarefa de associação induzida pelo contexto). Este estimador é o principal indicador do campo semântico em várias investigações sobre a memória semântica e tem se mostrado confiável e robusto (Steyvers, Shiffrin \& Nelson, 2004; Goodmon \& Nelson, 2004; Nelson \& Goodmon, 2002; Nelson, McKinney, Gee \& Janczura, 1998; Nelson, Schreiber \& McEvoy, 1992). O efeito da redução, induzido pelo contexto, deverá ser refletido no tamanho do conjunto das associadas, ou seja, a apresentação das categorias conceituais (palavras-alvo) na presença de diferentes contextos deverá produzir conjuntos menores de associadas do que quando os alvos são apresentados isoladamente. Isto acontecerá porque, na ausência de contexto, as associações são livres enquanto que, na presença de uma sentença, as palavras geradas devem partilhar o significado criado e restringido pela interação semântica entre as palavras de cada sentença e a sua estrutura sintática (Chomsky, 1957, 1965; Gazdar, Klein, Pullum \& Sag, 1985).
Além do conjunto de associadas, as normas puderam identificar, para diversas categorias, quais são as palavras mais freqüentes, quais as categorias que exibem maior índice de coocorrência de membros em diferentes condições, e as associadas mais provavelmente geradas independentemente das palavras-alvo serem disponibilizadas na ausência ou presença de contexto. A análise das associadas produzidas nas três condições possibilitou, ainda, verificar se o número de respostas corretas, as omissões, os erros de produção de palavras e as respostas idiossincráticas são influenciados pelo fato de as categorias conceituais serem experienciadas na presença ou não de contexto lingüístico.

\section{Método}

Participantes. Duzentos universitários, selecionados aleatoriamente, participaram do estudo voluntariamente.

Materiais. Vinte categorias semânticas amostradas de Janczura (1996) foram utilizadas: Artigo do Vestuário, Bebida Alcoólica, Calçado, Ciência, Cobra Venenosa, Emoção, Especialidade Médica, Ferramenta de Carpinteiro, Flor, Instrumento de Percussão, Jóia, Mobília, Modalidade de Ginástica, Raça de Cachorro, Regime de Governo, Ser Místico, Tempero, Tipo de Carne, Tipo de Dança e Tipo de Madeira. Foram elaboradas duas sentenças para cada categoria, perfazendo um total de 40 sentenças-alvo; cada uma explicitava um contexto diferente ${ }^{3}$. As sentenças eram compostas de 7 a 19 palavras (Média $=10,8, \mathrm{DP}=2,7$ ), e incluíam o nome de uma categoria semântica, que estava sublinhado, e um espaço em branco no qual os participantes deveriam escrever as respostas.

Procedimentos. Foi aplicada a tarefa de associação semântica que consistia na geração de palavras associadas para as sentenças-alvo. As instruções solicitavam que os participantes escrevessem, no espaço apropriado, o primeiro exemplo da categoria semântica que lhes viesse à mente, e que poderia completar a sentença no seu respectivo contexto. A técnica de solicitar a produção da primeira resposta (McEvoy e Nelson, 1982) teve por objetivo minimizar a possibilidade de efeitos de

\footnotetext{
${ }^{3}$ Em anexo encontram-se as descrições de algumas das categorias.
} 
encadeamento semântico (Nelson, McEvoy e Bajo, 1988) ou inibição na evocação (Raaijmakers e Shiffrin, 1981; Roediger, 1973). Cada participante produziu as associadas para 20 categorias diferentes em um dos dois contextos. O instrumento incluía 20 sentenças listadas randomicamente, apresentadas impressas em papel, em uma ordem aleatória, e continha duas folhas: a primeira disponibilizava as instruções da tarefa e, a segunda, as sentenças a serem completadas. Cada sentença foi respondida por 100 participantes. A sessão de coleta das normas de associação semântica iniciava-se com a apresentação das instruções gerais, objetivos e informações sobre a pesquisa, que foram fornecidas pelo experimentador, em voz alta. A seguir, os participantes eram instruídos a ler, silenciosamente, as instruções específicas da tarefa contidas na folha de rosto do instrumento. Após a leitura das instruções, os participantes começavam a completar as sentenças. O tempo para realização da tarefa era livre, mas os participantes levaram 15 minutos, em média, para terminá-la. Os participantes foram testados em pequenos grupos que variavam de 15 a 35 indivíduos, com as instruções da tarefa de produção lingüística : "Este estudo é sobre o que vem à mente das pessoas quando elas pensam em categorias. Por exemplo, você conhece a categoria tipos de música. Se eu lhe pedisse um membro desta categoria você poderia, citar "samba". A tarefa do participante é ler silenciosamente cada sentença e escrever o primeiro exemplo da categoria que lhe vier à mente e que se encaixe nela. Ilustrando, na sentença Um tipo de música muito popular nos anos 60 era Neste caso, a resposta poderia ser "rock-and-roll". Na folha seguinte, você encontrará várias sentenças. Escreva o primeiro exemplo que julgar se encaixar. NÃO deixe nenhuma sentença em branco.

\section{Resultados}

As normas de associação semântica para as 20 categorias contextualizadas podem ser observadas no anexo, junto com alguns exemplos. Apresentamse as seguintes medidas para cada categoria no Contexto I (CI) e Contexto II (CII): o tamanho da categoria (TC) que expressa o número de respostas diferentes e corretas excluindo-se as idiossincráticas (freqüência igual a 1, produzidas por somente 1 participante), o número total de respostas corretas excluindo-se as idiossincráticas (C), o número de omissões $(\mathrm{O})$ ou respostas em branco, o número de erradas (E), e o de idiossincráticas (I). Além destas medidas, cada categoria incluiu as respostas produzidas e as suas respectivas porcentagens de ocorrência na amostra. Cada resposta produzida em CI e CII foi avaliada por 3 juízes independentes, sendo consideradas como corretas as julgadas como apropriadas para a categoria no contexto considerado por, pelo menos, 2 juízes. Foram categorizados como o mesmo item os sinônimos, variações de número e gênero desde que não implicassem em diferenças semânticas evidentes.

A média de respostas corretas produzidas por categoria em CI foi 90,1 (DP = 8,0), e em CII foi 85,2 $(\mathrm{DP}=9,4)$. Um teste T para amostras independentes evidenciou a inexistência de diferença significativa entre as médias ( $\mathrm{t}=1,779, \mathrm{gl}=38, \mathrm{p}=0,08$ ) o que significa que, independentemente do contexto semântico em que a categoria foi apresentada, os participantes tenderam a produzir um número semelhante de acertos. Este resultado chama a atenção considerando que CI e CII são muito distintos semanticamente.

O tamanho da categoria, erros, omissões e respostas idiossincráticas foi contrastado entre as condições Isolada (associadas coletadas na ausência de contexto) versus Contextualizada (associadas coletadas na presença de CI ou CII). As medidas da condição Isolada foram calculadas utilizando-se as normas de Janczura (1996). Naquele estudo, as associadas de 69 categorias semânticas foram coletadas utilizando-se a técnica de associação livre que solicitou somente a primeira associada para cada categoria-alvo. Os procedimentos utilizados na coleta das associadas do presente estudo são idênticos àqueles do estudo citado, excetuando que as categorias, neste estudo, foram apresentadas no contexto de uma sentença.

$\mathrm{Na}$ condição Isolada foram observadas as seguintes medidas: tamanho da categoria $=14,25$ (DP $=4,39)$, erros $=14,70(\mathrm{DP}=9,89)$, omissões $=1,90$ $(\mathrm{DP}=3,37)$ e respostas idiossincráticas $=3,55(\mathrm{DP}=$ 2,29); em CI o tamanho da categoria $=7,50(\mathrm{DP}=$ $3,35)$, erros $=4,35(\mathrm{DP}=4,08)$, omissões $=1,15$ (DP $=1,79)$, e idiossincráticas $=3,7(\mathrm{DP}=2,90)$; em CII as medidas foram: tamanho da categoria $=7,75$ (DP 
$=2,97)$, erros $=9,15(\mathrm{DP}=8,24)$, omissões $=0,45$ ( $\mathrm{DP}=1,15)$, e idiossincráticas $=4,35(\mathrm{DP}=2,54)$.

Análises da variância revelaram que o fator Condição de Apresentação da Categoria (Isolada, CI, CII) produziu efeitos significativos no tamanho da categoria $[\mathrm{F}(2,57)=22,37, \mathrm{MS}=13,096, \mathrm{p}=0,000]$, e no número de erros $[\mathrm{F}(2,57)=8,826$, MS $=60,795$, $p=0,000]$. Testes subseqüentes para as diferenças entre as médias (LSD, $\mathrm{p}=0,05$ ) indicaram que foram significativas as diferenças entre as médias do tamanho da categoria entre as condições Isolada versus CI e Isolada versus CII; também foram significativas as diferenças entre as médias de erros nas mesmas comparações. A ANOVA para as respostas idiossincráticas não registrou efeito significativo deste fator $[\mathrm{F}(2,57)=0,537, \mathrm{MS}=6,705]$.

Os resultados revelam que o contexto reduz o número de associadas produzidas na tarefa de associação semântica e que esta diminuição é, aproximadamente, de 50\%. Destaca-se que a magnitude da redução é independente do contexto apresentado. Além disto, são observados mais erros de associação semântica quando as categorias são apresentadas isoladamente. Por outro lado, o número médio de respostas idiossincráticas é pequeno e não é influenciado pelo fato da categoria ter sido apresentada isoladamente ou em dois contextos diferentes.

Uma análise da primeira associada de cada categoria - aquela que está mais fortemente associada ao conceito - mostrou que estas palavras são mais fortemente associadas à categoria quando apresentadas contextualizadas, $[\mathrm{F}(2,57)=6,630$, MS $=242,839, p=0,003]$. As médias observadas em cada condição foram 29,9 (DP = 7,97), 44,9 (DP = 21,15), e $46,1(\mathrm{DP}=14,76)$ para as condições isolada, CI e CII, respectivamente. Testes subseqüentes para verificar diferenças entre as médias (LSD, $p=0,05$ ) apontaram diferenças significativas entre Isolada versus CI, e Isolada versus CII.

O contraste entre as duas bases de dados permitiu gerar uma medida de ocorrência intracategórica de respostas entre as três condições. A medida viabilizou identificar quais as categorias mais “comuns”, isto é, aquelas cujos membros tendem a ser lembrados com maior probabilidade, independente de a categoria ter sido apresentada isolada ou contextualizada. Além disto, identificaram-se quais os membros mais “populares”. O índice de ocorrência intra-categórica foi calculado através da equação:

$$
C_{a}=\frac{\sum p_{k}}{n}
$$

onde: $C_{a}$ corresponde ao índice de ocorrência intra-categórica da categoria $a$; $p$ corresponde ao número de ocorrências intra-categorias para cada membro gerado entre as três condições que varia de 1 a 3 (isto é, se o membro $k$ foi produzido apenas em uma das condições atribuiu-se o valor 1 , se apareceu em duas condições o valor 2 e, a três condições o valor 3 ); $k$ corresponde ao membro da categoria $a$; e, $n$ corresponde ao número total de itens diferentes gerados para a categoria considerando as três condições. A Tabela 1, a seguir, apresenta os membros das categorias que foram produzidos nas três condições em cada categoria, e o índice de ocorrência intracategórica $\left(C_{a}\right)$.

Tabela 1. Membros mais freqüentes e índice de ocorrência intra-categórica $\left(C_{a}\right)$

\begin{tabular}{llc}
\hline \multicolumn{1}{c}{ Categoria } & \multicolumn{1}{c}{ Membros } & $C_{a}$ \\
\hline Cobra Venenosa & cascavel, coral, jararaca, sucuri, & 2,22 \\
Flor & naja & 1,93 \\
Regime de Governo & republicano, democrático & 1,93 \\
Instrumento de Percussão & bateria, tambor, bumbo, tamborim & 1,92 \\
Calçado & tênis, sapato, bota & 1,88 \\
Jóia & anel, colar, brinco, pérola, & 1,83 \\
& brilhante & \\
Tipo e Madeira & pau-brasil, mogno, jacarandá, ipê, & 1,83 \\
Bebida Alcoólica & cerejeira, cedro & 1,73 \\
Ferramenta de Carpinteiro & cerveja, vinho & 1,71 \\
Mobília & serrote, plaina, martelo, machado, \\
Ciência & sofmáo cadeira, mesa & 1,63 \\
Especialidade Médica & Biologia, física, medicina, & 1,59 \\
Emoção & psicologia & 1,53 \\
Artigo do Vestuário & Psiquiatria & 1,52 \\
Tempero & boro, desespero, dor, tristeza \\
Modalidade de Ginástica & bermuda, shorts & 1,48 \\
Raça de Cachorro & natação, olímpica, rítmica & 1,40 \\
Tipo de Dança & doberman, pastor alemão & 1,38 \\
Tipo de Carne & --- & 1,36 \\
Ser Místico & Vermelha & 1,22 \\
\hline & --- & 1,20 \\
& & 1,08 \\
\hline
\end{tabular}

\section{Contexto e normas para palavras}

A tabela acima ordena as categorias a partir daquela que apresenta maior índice de ocorrência intra-categórica até aquela com menor índice. O número de itens comuns nas três condições variou de 0 a 6. A categoria semântica com maior índice de ocor- 


\section{Gerson Américo Janczura}

rência intra-categórica foi Cobra Venenosa e, para duas categorias (Tipo de Dança e Ser Místico), nenhum membro comum foi citado nas três condições.

Observando $C_{a}$ e o número de itens comuns identificados nas três condições (itens “populares”), verificou-se que os dois parâmetros estão forte e positivamente associados ( $\mathrm{r}=0,71, \mathrm{p}=0,001$, bi-caudal), ou seja, na medida em que o índice de ocorrência intra-categórica eleva-se também constata-se que o número de itens populares é maior. Entretanto, esta relação é inversa quando $C_{a}$ é comparado com o número total de respostas diferentes produzidas nas três condições ( $r=-0,87, p=0,05$, bi-caudal). Neste caso, quanto maior for o número de respostas diferentes nas três condições, simultaneamente, tanto menor o índice de ocorrência intra-categórico. Por fim, constatou-se que a quantidade de itens populares está inversamente correlacionada com o número de respostas diferentes nas três condições ( $r=-0,51, p=0,05$, bi-caudal), ou seja, categorias que produziram um número maior de respostas diferentes nas três condições obtiveram um número menor de itens populares.

\section{Considerações finais}

As normas de associação semântica para categorias conceituais permitiram estimar a magnitude da redução de seu campo semântico. Quando os indivíduos são solicitados a produzir membros de categorias contextualizadas, observa-se que o número médio de associadas semânticas é cinqüenta por cento menor comparando-se à mesma categoria se apresentada sem qualquer restrição associativa. Apesar do contexto manipulado constituir-se de sentenças simples, é razoável esperar que uma redução também ocorreria com materiais mais complexos como parágrafos, textos, diálogos ou cenas. Neste sentido, as normas aqui coletadas geram uma expectativa da dimensão da restrição associativa com outros tipos de contextos. Evidentemente, podem ser propostos contextos que variam desde extremamente restritivos semanticamente até situações que permitam maior amplitude associativa.

Destaca-se, também, que a interação entre categorias e contextos promove o estabelecimento associações mais fortes com a primeira associada quando comparada à situações onde a categoria é apresentada isoladamente. Este resultado pode ser explicado pelo fato do contexto reduzir as possibilidades de associações que se encaixam na semântica do mesmo.

A redução do campo semântico tem uma outra conseqüência interessante: o número de erros cometidos na ausência de contexto é maior do que em sua presença. Isto pode parecer contra-intuitivo: não seria esperado que, na ausência de restrições contextuais, os indivíduos tivessem mais liberdade para produzir associadas aceitáveis? Na verdade, o contexto parece promover o estabelecimento de critérios de aceitabilidade que auxiliam o indivíduo a estabelecer o campo semântico pertinente produzindo, assim, um número menor de erros de associação.

O contraste entre diferentes normas evidenciou que, independentemente das categorias serem apresentadas isoladamente ou contextualizadas, na maioria das categorias utilizadas existem alguns membros que são mais provavelmente evocados da memória. Além disto, as categorias variam na quantidade deste tipo de membros. Os resultados mostraram que categorias que apresentam um número grande de associados tendem a incluir um número menor de membros "populares". Além disto, se for considerado o número total de membros diferentes gerados nas condições investigadas, verifica-se que categorias com maior variabilidade estão associadas com índices menores de membros que ocorrem simultaneamente em diferentes contextos ou isoladamente.

Em resumo, o contexto provoca a redução do campo semântico revelado pelo número de associadas, reduz o número de erros associativos, e gera um primeiro membro fortemente associado à categoria. Por fim, as categorias mais enxutas tendem a produzir um número maior de membros "populares”.

\section{Referências Bibliográficas}

Balota, D.A., Ferraro, F.R., \& Connor, L.T. (1991). On the early influence of meaning in word recognition: A review of the literature. Em P. J. Schwanenflugel (Org.), The psychology of word meaning. Hillsdale, NJ: LEA.

Barsalou, L.W. (1992). Cognitive psychology: An overview for cognitive scientists. Lawrence Erlbaum Associates: New Jersey. 
Battig, W.F. \& Montague, W.E. (1969). Category norms for verbal items in 56 categories: A replication and extension of the Connecticut category norms. Journal of Experimental Psychology Monograph, 80 (3, part 2).

Bishop, D.V.M. \& Robson, J. (1989). Umimpaired short-term memory and rhyme judgment in congenitally speechless individuals: Implications for the notion of "articulatory coding”. Quarterly Journal of Experimental Psychology, 41A, 123140.

Callejas, A., Correa, A., Lupiáñez, J. \& Tudela, P. (2003). Normas asociativas intracategoriales para 612 palabras de seis categorias semânticas en Español. Psicológica, 24, 185-214.

Chomsky, N. (1957). Syntactic structures. The Hague: Mouton.

Chomsky, N. (1965). Aspects of a theory of syntax. Cambridge, MA: MIT Press.

Eling, P. (1983). Consistency of ear advantage: An improvement due to increase in presentation rate. Neuropsychologica, 21, 419-423.

Elmes, D. G., Dye, C. J. \& Herdelin, N. J. (1983). What is the role of affect in the spacing effect? Memory \& Cognition, 11, 144-151.

Gazdar, G., Klein, E., Pullum, G. \& Sag, I. (1985). Generalized phrase structure grammar. Oxforf $>$ Basil Blackwell.

Goodmon, L. B. \& Nelson, D. L. (2004). Strengthening the activation of unconsciously activated memories. Memory and Cognition, 32, 804-818.

Hampton J.A. \& Gardiner, M. M. (1983). Measures of internal category structure: A correlational analysis of normative data. British Journal of Psychology, 74, 491-516.

Hirsh, K. W. \& Tree, J. J. (2001). Word association norms for two cohorts of British adults. Journal of Neurolinguistics, 14, 1-44.

Hulme, C., Roodenvys, S., Schweickert, R., Brown, G. D. A., Martin, S. \& Stuart, G. (1997). Wordfrequency effects on short-term memory tasks: Evidence for a redintegration process in immediate serial recall. Journal of Experimental Psychology: Learning, Memory, and Cognition, 23, 1217-1232.

Jacoby, L. L. \& Dallas, M. (1981). On the relationship between autobiographical memory and perceptual learning. Journal of Experimental. Psychology: General, 110, 306-340.

Janczura, G. A. \& Nelson, D. L. (1999). Concept accessibility as the determinant of typicality judgments. American Journal of Psychology, 112, 1-19.

Janczura, G. A. (1996). Normas associativas para 69 categorias semânticas. Psicologia: Teoria e Pesquisa, 12, 237-244.

Kozlowsky, L. T. (1977). Effects of distorted auditory and rhyming cues on retrieval of tip-of-tongue words by poets and nonpoets. Memory \& Cognition, 5, 477-481.

Kucera, H. \& Francis, W. N. (1967). Computational analysis of present-day American English. Providence, RI: Brown University Press.

Libkuman, T. M. (1994). Norms for words that rhyme. Behavior Research Methods, Instrument, \& Computers, 26, 278-322.

Lupiáñes, J., Rueda, M. R., Ruz, M. \& Tudela, P. (2000). Processing of attended and ignored parafoveal words: semantic processing and inhibition. Psicológica, 21, 233-257.

Macizo, P., Gómez-Ariza, C. J. \& Bajo, M. T. (2000). Associative norms of 58 Spanish words for children from 8 to 13 years old. Psicológica, 21, 287-300.

Marques, J. F. (1997). Normas de tipicidade e familiaridade para diferentes categorias de itens verbais. Revista Portuguesa de Psicologia, 32, 3555.

McEvoy, C. L. \& Nelson, D. L. (1982). Category name and instance norms for 106 categories of various sizes. American Journal of Psychology, 95, 581-634.

Nelson, D. L. \& Goodmon, L. B. (2002). Experiencing a word can prime its accessibility and its 


\section{Gerson Américo Janczura}

associative connections to related words. Memory and Cognition, 30, 380-398.

Nelson, D. L., McEvoy, C. L. \& Bajo, M. T. (1984). Retrieval processes in perceptual recognition and cued recall: The influence of category size. Memory \& Cognition, 12, 498-506.

Nelson, D.L., McEvoy, C.L. \& Bajo, M.T. (1988). Lexical and semantic search in cued recall, fragment completion, perceptual identification, and recognition. American Journal of Psychology, 101, 465-480.

Nelson, D.L., McEvoy, C.L. \& Schreiber, T.A. (1999). The University of South Florida Word Association, Rhyme and Fragment Norms. http://www.usf.edu/ FreeAssociation/.

Nelson, D. L., McKinney, V. M., Gee, N. R., \& Janczura, G. A. (1998). Interpreting the influence of implicitly activated memories on recall and recognition. Psychological Review, 105, 299324.

Nelson, D. L., Schreiber, T. A \& McEvoy, C. L. (1992). Processing implicit and explicit representations. Psychological Review, 99, 322-348.

Paivio, A., Yuille, J. C. \& e Madigan, S. A. (1968). Concreteness, imagery, and meaningfulness values for 925 nouns. Journal of experimental Psychology: Monograpgh Supplement, 76 (1Part 2), 1-25.

Pérez, M. A. \& Navalón, C. (2003). Normas españolas de 290 nuevos dibujos: Acuerdo em la denominación, concordancia de la imagen, familiaridad, complexidad visual y variabilidad de la imagen. Psicológica, 24, 215-241.

Pinheiro, A. M. V. (1996). Contagem de freqüência de ocorrência de palavras expostas a crianças na faixa pré-escolar e séries iniciais do primeiro grau. Associação Brasileira de Dislexia, São Paulo.

Pompéia, S., Paes, A. T. \& Bueno, O. F. A. (2003). Teste de completação de letras: Estímulos para uso no Brasil. Psicologia: Teoria e Pesquisa, 19, 65-73.
Raaijmakers, J.G.W. \& Shiffrin, R.M. (1981). Search of associative memory. Psychological Review, 88, 93-134.

Roediger, H. L. (1973). Inhibition in recall from cuing with recall targets. Journal of Verbal Learning and Verbal Behavior, 12, 644-657.

Sardinha, T. B. (2004). Lingüística de Corpus. Barueri, SP: Manole.

Shaw, R. J. \& Craik, F. I. M. (1989). Age differences in predictions and performance on a cued recall task. Psychology \& Aging, 4, 131-135.

Snodgrass, J.G. \& Vanderwart, M. (1980). A standardized set of 260 pictures: Norms for name agreement, familiarity, and visual complexity. Journal of Experimental Psychology: Human Learning and Memory, 6, 174-215.

Stadler, M. A., Roediger, H. L. \& McDermott, K. B. (1999). Norms for word lists that create false memories. Memory and Cognition, 27, 494-500.

Stein, L. M. \& Pergher, G. K. (2001). Criando falsas memórias em adultos por meio de palavras associadas. Psicologia: Reflexão e Crítica, 14, 353366.

Steyvers, M., Shiffrin, R. M. \& Nelson, D. L. (2004) Word Association Spaces for Predicting Semantic Similarity Effects in Episodic Memory, Em A. F. Healy(Ed), Experimental cognitive psychology and its applications. Washington, DC, US: American Psychological Association.

Thorndike, E. L. \& Lorge, I. (1944). The teachers word book of 30,000 words. New York: Columbia University, Teachers College, Bureau of Publications.

Wiegel-Crump, C. A. \& Dennis, M. (1986). Development of word finding. Brain \& Language, 27, 1-23.

O autor agradece o apoio do CNPq para a realização deste projeto.

Agradecimento ao Prof. Galber J. O. Maciel pelas sugestões para o aprimoramento deste trabalho. 


\section{Anexo}

Normas associativas para categorias semânticas contextualizadas, onde: $\mathrm{CI}=$ Contexto I, CII = Contexto II, TC = Tamanho da Categoria, $\mathrm{C}=$ Número de respostas corretas, $\mathrm{O}=$ Omissões, $\mathrm{E}=$ Erros, $\mathrm{I}=$ Idiossincráticas).

\section{Artigo do Vestuário}

$\mathrm{CI}=\mathrm{Um}$ artigo do vestuário frequentemente usado para ir ao shopping é _ _ (TC $=12, \mathrm{C}=94, \mathrm{O}=0, \mathrm{E}$ $=1, \mathrm{I}=5$ ). Calça $38 \%$, Jeans $18 \%$, Bermuda $7 \%$, Camiseta 6\%, Blusa 5\%, Vestido 4\%, Saia 4\%, Camisa 3\%, Sapato 3\%, Boné 2\%, Bolsa 2\%, Short 2\%.

$\mathrm{CII}=\mathrm{Um}$ artigo do vestuário frequentemente usado para ir à praia é _ $(\mathrm{TC}=7, \mathrm{C}=93, \mathrm{O}=0, \mathrm{E}=3$, I =4). Biquíni 60\%, Sunga 12\%, Maiô 9\%, Bermuda $5 \%$, Short de Banho 3\%, Roupa de Banho 2\%, Short $2 \%$.

\section{Bebida Alcoólica}

$\mathrm{CI}=$ __ é uma bebida alcoólica servida em comemorações (TC $=4, \mathrm{C}=98, \mathrm{O}=0, \mathrm{E}=1, \mathrm{I}=1$ ). Champagne 56\%, Cerveja 22\%, Vinho 18\%, Caipirinha $2 \%$.

$\mathrm{CII}=\ldots$ é uma bebida alcoólica preferida em países do Leste Europeu (TC = 5, C = 95, O = 0, E = 2, I = 3). Vodka 36\%, Whiskey 20\%, Vinho 20\%, Cerveja $16 \%$, Pinga $3 \%$.

\section{Ciência}

$\mathrm{CI}=$ __ é uma das ciências que mais avançou recentemente. ( $\mathrm{TC}=8, \mathrm{C}=89, \mathrm{O}=1, \mathrm{E}=5, \mathrm{I}=5$ ). Informática 28\%, Psicologia 19\%, Medicina 15\%, Genética 12\%, Biologia 5\%, Física 4\%, Química 4\%, Biogenética $2 \%$.

CII = Atualmente, a ciência que está desafiando os valores morais, religiosos e éticos da Igreja Católica é __ (TC = 9, C = 81, O = 0, E = 12, I = 7). Genética $22 \%$, Biologia 19\%, Psicologia 10\%, Física 10\%, Medicina 6\%, Sociologia 5\%, Teologia 4\%, Filosofia 3\%, História 2\%.

\section{Emoção}

CI = Saber do falecimento do avô provocou em Juca a emoção de __ (TC = 7, C = 91, O = 0, E = 5, I = 4). Tristeza 46\%, Chorar 16\%, Perda 12\%, Dor 9\%, Desespero 4\%, Felicidade 2\%, Saudade 2\%.
$\mathrm{CII}=$ Sofrer um acidente de automóvel provocou em Juca a emoção de _ $(\mathrm{TC}=13, \mathrm{C}=74, \mathrm{O}=0, \mathrm{E}=$ 22 , I = 4). Medo 29\%, Chorar 12\%, Dor 5\%, Tristeza $4 \%$, Pavor 4\%, Trauma 3\%, Choque 3\%, Desespero $3 \%$, Susto $3 \%$, Ansiedade $2 \%$, Angústia 2\%, Pânico $2 \%$, Sofrer $2 \%$.

\section{Especialidade Médica}

$\mathrm{CI}=\mathrm{A}$ especialidade médica recomendada nos casos de perda dos sentidos é _ $(\mathrm{TC}=6, \mathrm{C}=65, \mathrm{O}=$ $3, \mathrm{E}=16, \mathrm{I}=1$ ). Neurologia 25\%, Clínica Geral 18\%, Psiquiatria 10\%, Fisioterapia 7\%, Clínica Médica 3\%, Cirurgião 2\%.

$\mathrm{CII}=\mathrm{A}$ especialidade médica recomendada nos casos de paralisia cerebral é _ $(\mathrm{TC}=6, \mathrm{C}=83, \mathrm{O}=$ $5, \mathrm{E}=11, \mathrm{I}=2$ ). Neurologia $62 \%$, Psiquiatria $6 \%$, Neurocirurgia 5\%, Fisioterapia 4\%, Cirurgia 3\%, Cardiologia 3\%.

\section{Regime de Governo}

$\mathrm{CI}=$ Daniel sempre lutou contra o regime de governo $(\mathrm{TC}=12, \mathrm{C}=92, \mathrm{O}=1, \mathrm{E}=5, \mathrm{I}=2)$. Ditatorial 18\%, Autoritário 17\%, Militar 12\%, Comunista $11 \%$, Monárquico 11\%, Socialista 5\%, Democrático 5\%, Republicano 2\%, Parlamentarista 2\%, Capitalista 2\%, Imperial 2\%.

$\mathrm{CII}=\mathrm{O}$ regime de governo preferido pelos brasileiros é _ ( $\mathrm{TC}=4, \mathrm{C}=93, \mathrm{O}=0, \mathrm{E}=3, \mathrm{I}=4)$. Democracia 46\%, Presidencialismo 37\%, Republicano $8 \%$, Anarquia $2 \%$.

\section{Ser Místico}

CI = Um ser místico típico de religiões afro-brasileiras é __ ( TC $=11, \mathrm{C}=77, \mathrm{O}=2, \mathrm{E}=11, \mathrm{I}=10)$. Pai-de-Santo 22\%, Iemanjá 15\%, Saci Pererê 11\%, Orixá $6 \%$, Ogum $5 \%$, Preto Velho 5\%, Oxum $4 \%$, Oxalá 3\%, Exu 2\%, Caboclo 2\%, Zumbi 2\%.

$\mathrm{CII}=$ Os gregos adoravam __ como o ser místico mais sagrado ( $\mathrm{TC}=7, \mathrm{C}=71, \mathrm{O}=1, \mathrm{E}=24, \mathrm{I}=4$ ). Zeus 57\%, Hércules 3\%, Eros 3\%, Deus 2\%, Vênus $2 \%$, Deuses 2\%, Afrodite $2 \%$. 